LA GESTIÓN DEL CONOCIMIENTO EN LAS MIPYMES: CASO DE UNA CONSULTORA EN YUCATÁN, MÉXICO.

\title{
La Gestión del Conocimiento en las Mipymes: Caso de una Consultora en Yucatán, México
}

\section{Knowledge management in MSMEs: The case of a Consulting Office in Yucatan, Mexico}

\author{
Ángela Guadalupe Manzano Santana*, Jennifer Mul Encalada**
}

\begin{abstract}
*Estudiante de la maestría en Gestión y Cambio Organizacional. Universidad Autónoma de Yucatán, Facultad de Contaduría y Administración. México. ORCID: https://orcid.org/0000-0003-4200-8679.

**Doctora en Ciencias de la Administración. Universidad Autónoma de Yucatán, Facultad de Contaduría y Administración. México. Email: jeni.mul@correo.uady.mx. ORCID: http://orcid.org/0000-0003-0034-8165.
\end{abstract}

Correo para recibir correspondencia: ange_manzano2311@hotmail.com 
LA GESTIÓN DEL CONOCIMIENTO EN LAS MIPYMES: CASO DE UNA CONSULTORA EN YUCATÁN, MÉXICO.

\section{RESUMEN}

OBJETIVO: Analizar los procesos de gestión del conocimiento en una organización que presta servicios profesionales de consultoría en la ciudad de Mérida, Yucatán, México.

MATERIAL Y MÉTODO: Este trabajo se realizó con un enfoque cualitativo mediante el estudio de caso. Para la obtención de la información se realizaron entrevistas semiestructuradas al personal de la organización, así como la revisión documental y observación.

RESULTADOS: La organización ha trabajado más en prácticas de adquisición de conocimiento y menos en las de compartición y protección. La principal contribución radica en la comprobación empírica de cómo se dan los procesos de gestión del conocimiento en una pequeña empresa y la manera en cómo éstos afectan sus resultados.

CONCLUSIONES: Las estrategias recomendadas proponen evitar la rotación del personal, mantener el orden y codificación de la información, desarrollar manuales, establecer mecanismos formales de reconocimiento, obtener un acceso controlado a la información, entre otras.

PALABRAS CLAVE: Gestión del conocimiento. Mipymes. Organización. Aprendizaje.

\section{ABSTRACT}

OBJECTIVE: To analyze knowledge management processes in an organization dedicated to professional consulting services in Merida, Yucatán; Mexico.

MATERIAL AND METHOD: This is a qualitative work carried out through the study case technique. A semi-structured questionnaire was used for the conducted interviews to personnel from the organization. Other techniques were also implemented for collecting information: documentary review and observation. 
LA GESTIÓN DEL CONOCIMIENTO EN LAS MIPYMES: CASO DE UNA CONSULTORA EN YUCATÁN, MÉXICO.

RESULTS: The organization has worked more on knowledge acquisition practices and less on sharing and protection. The main contribution lies in the empirical verification on how knowledge management processes occur in a small company and how they affect its results.

CONCLUSIONS: The recommended strategies that are proposed are the following: avoiding staff turnover, maintaining order and coding of information, developing manuals, establishing formal recognition mechanisms, obtaining controlled access to information, among others.

KEYWORDS: Knowledge management. MSMEs. Organization. Learning.

\section{INTRODUCCIÓN}

Diversos autores coinciden en que el conocimiento es el principal recurso de la economía del futuro y que el proceso más importante para el desarrollo económico es el aprendizaje (Arboníes, 2006; Sánchez y Ríos, 2011). En este contexto, la Gestión del Conocimiento (GC) cobra mayor importancia. Estudios empíricos se han enfocado al estudio de esta gestión en grandes organizaciones de tecnología o innovadoras y existen menos que presentan evidencia de su presencia en las micro, pequeñas y medianas empresas (Mipymes). Sin embargo, se ha demostrado que las Mipymes pueden estar en condiciones de incrementar de manera notoria su crecimiento y sus ventajas competitivas a través de una adecuada GC (Maldonado, Martínez y García, 2012).

Es importante mencionar que, a diferencia de la Unión Europea, en América Latina no hay un acuerdo sobre la definición de Mipyme, ya que no existe un mercado común ni una real integración económica en países con distintos tamaños empresariales (Dini y Stumpo, 2018). En México, las micro, pequeñas y medianas empresas del sector industria son aquellas que cuentan con un personal de 0 a 250, y con un rango de ventas anuales de hasta 250 millones de pesos. Las que corresponden al sector comercio, tienen de 0 a 100 personas laborando en ellas y alcanzan a generar 250 millones de pesos anuales en ventas. Por último, las Mipymes que realizan actividades de servicios, son aquellas que tienen un personal de 0 a 100 trabajadores y ventas anuales de hasta 250 millones de pesos (DOF, 2009). 
LA GESTIÓN DEL CONOCIMIENTO EN LAS MIPYMES: CASO DE UNA CONSULTORA EN YUCATÁN, MÉXICO.

La relevancia que tiene el estudio de la GC en las Mipymes se debe al alto porcentaje de participación con el que cuentan tanto en México como en las diversas economías internacionales. Representan aproximadamente el 90\% del sector empresarial en todo el mundo (Durán, 2017). De acuerdo con el Instituto Nacional de Estadística y Geografía (INEGI), en México, las Mipymes cuentan con una participación de más del 99\% y un porcentaje de empleo del 68.4\% de la totalidad de unidades económicas (INEGI, 2019).

Para hacer frente a la nueva economía, las Mipymes requieren implementar la gestión del conocimiento; sin embargo, ello implica también encontrarse ante importantes retos los cuales deben poder superar para lograr su adaptación y permanecer en el tiempo. Los beneficios al incorporar una gestión basada en el conocimiento pueden ayudarlas a crecer y ser más innovadoras. Podrían lograr permanencia, posicionamiento y apertura en el mercado existente y nuevos; colaboración entre los miembros de la organización para el cumplimiento de sus funciones y reducción de la duplicidad de actividades; desarrollo de nuevos productos; una diferenciación comercial; respuesta oportuna a sus stakeholders; mejorar el desempeño financiero; por mencionar los principales.

El presente trabajo, analiza el caso de una organización de tamaño pequeño que ofrece servicios profesionales de consultoría contable, fiscal, administrativa y financiera a empresas en la ciudad de Mérida, Yucatán.

Actualmente la consultora presenta algunas deficiencias en cuanto a su comunicación interna, compartición de conocimiento y falta de claridad de las actividades que desempeñan los colaboradores. Ante esta situación y debido a que los directivos desean establecer estrategias para mejorar su posición en el mercado mediante la diferenciación e innovación, realizar un estudio sobre la gestión del conocimiento le permitiría conocer su situación y áreas de oportunidad.

Con base en lo anterior, el presente trabajo plantea la siguiente pregunta de investigación: ¿Cuál es la situación que guarda la gestión del conocimiento en una organización que presta servicios profesionales de consultoría en la ciudad de Mérida, Yucatán? A partir de dicha pregunta se estableció como objetivo general, analizar procesos de gestión del conocimiento en una organización que presta servicios profesionales de consultoría en la ciudad de Mérida, Yucatán, México, con la finalidad de identificar áreas de oportunidad para mejorar dicha gestión. 
LA GESTIÓN DEL CONOCIMIENTO EN LAS MIPYMES: CASO DE UNA CONSULTORA EN YUCATÁN, MÉXICO.

\section{Gestión del conocimiento y sus dimensiones}

Para Davenport y Prusak (2000) el conocimiento es una mezcla de experiencia enmarcada, valores, información contextual y conocimiento experto, misma que proporciona un marco para evaluar e incorporar nuevas experiencias e información y que se origina y aplica en la mente de los conocedores. En las organizaciones es integrado en documentos, repositorios, rutinas, procesos, prácticas y normas. Existen diversas clasificaciones para los tipos de conocimiento. Nonaka (1994) se refiere a dos en particular, el conocimiento tácito y el explícito, el primero es aquél que tiene cualidad personal por lo que es más difícil formalizar y comunicar, el segundo es aquél que es fácilmente transmisible en conocimiento formal, por lo que se captura en registros del pasado, como bibliotecas, archivos y bases de datos. Pérez-Montoro (2018) señala que, el conocimiento puede ser dividido en seis tipos, los cuales se agrupan en tres parejas: tácito/explícito, individual/organizacional, interno/externo.

Para Rodríguez (2006), la gestión del conocimiento consiste en un conjunto de procesos sistemáticos que se encuentran orientados al desarrollo de la organización y/o personal y, por consiguiente, a la generación de una ventaja competitiva, ya sea para esta organización y/o el individuo. Para el presente trabajo, se considerará como gestión del conocimiento "el proceso que se encarga del diseño e implementación de prácticas para la adquisición, almacenamiento, compartición, aplicación y protección del conocimiento, con el objeto de mejorar la capacidad de las organizaciones para la resolución de problemas, la innovación y contribuir a la consecución de sus ventajas competitivas" (Mul y Ojeda, 2014, p. 6).

Existen diferentes modelos de gestión de conocimiento que sirven como marco de referencia para comprender dicho proceso, algunos ejemplos de estos modelos son los de Wiig (1993), Nonaka y Takeuchi (1995), Kerschberg (2001), Riesco (2004), Angulo y Negrón (2008), entre otros. Aunque cada uno de estos autores propone diversas dimensiones, existen similitudes entre ellas. La mayoría de las propuestas coinciden en cinco: adquisición, almacenamiento, compartición, aplicación y protección (Mul y Ojeda, 2014).

La adquisición puede entenderse como la aprehensión cognoscitiva que tienen los miembros de las organizaciones, del conocimiento que se ha compartido. Riesco (2004) menciona que, para la adquisición pueden utilizarse dos estrategias a) compra o alquiler del conocimiento en el entorno del mercado o de las personas externas a la organización b) creación de conocimiento 
LA GESTIÓN DEL CONOCIMIENTO EN LAS MIPYMES: CASO DE UNA CONSULTORA EN YUCATÁN, MÉXICO.

dentro de la misma organización. Kerschberg (2001), amplía más este punto al exponer que durante la adquisición, es recomendable capturar el conocimiento originado por los expertos en un dominio, esto puede ser a través de entrevistas, estudios de caso y otras técnicas.

Con respecto al almacenamiento, Riesco (2004) menciona que este debe ser filtrado de manera que se disponga únicamente del conocimiento valioso y necesario. Esta clasificación requiere de una codificación que varía según diversos criterios, por ejemplo, los distintos tipos de dominio o áreas especializadas de conocimiento, el grado de accesibilidad y disponibilidad de la información, la disposición de un lenguaje común, el apoyo de las TIC, su finalidad y uso, su nivel de estructuración, entre otras. En ese sentido, se trata de una serie de prácticas para retener y guardar el conocimiento en el tiempo y que pueda ser recuperado posteriormente para su uso en la organización.

En el compartimento, se pretende que el conocimiento involucrado en la organización llegue mediante su transferencia y su difusión a los miembros de la comunidad que lo necesiten (Pérez-Montoro, 2008). Para el caso del conocimiento explícito y del tácito explicable, ésta propagación se realiza a través de documentos, bases de datos o cualquier otro medio físico o virtual, en el caso del tácito no explicable se realiza a través de diversas estrategias de socialización entre el individuo que posee el conocimiento y el resto de la comunidad interesada en él. El modelo holístico para la gestión del conocimiento de Angulo y Negrón (2008), denomina a esta fase como difusión, el cual es un elemento fundamental para el proceso productivo y un componente esencial para el bien personal. Las personas necesitan construir conocimientos que den oportunidad a lograr respuestas más adecuadas antes las circunstancias que se presentan en la vida cotidiana, por lo que deben disponer de mecanismos que les permita transferir esa información y sin los cuales no se podría elaborar el conocimiento.

Wiig (2004) menciona que, al usar (aplicar) el conocimiento, éste se convierte en la base para un mayor aprendizaje e innovación. La aplicación es considerada como prácticas que van encaminadas a la utilización del conocimiento, las cuales impactan en el desarrollo de nuevos productos y mejora de procesos. Caraballo, Mesa y Herrera (2009), sugieren el uso de plataformas de conocimientos como las intranets, portales, escenarios, entre otras herramientas para incentivar a los colaboradores a consumir información que incremente su conocimiento. 
LA GESTIÓN DEL CONOCIMIENTO EN LAS MIPYMES: CASO DE UNA CONSULTORA EN YUCATÁN, MÉXICO.

Kerschberg (2001) recomienda el establecimiento de controles de acceso y políticas de seguridad para proteger la base de conocimiento y la propiedad intelectual. Cuando se decide preservar un activo de conocimiento, se acostumbra a realizar su protección legal con el fin de continuar disfrutando de los beneficios que ofrece. De igual manera, Ramírez, Mercado y Barbosa (2012), mencionan que los derechos de propiedad intelectual constituyen un instrumento importante con el cual las economías que generan conocimiento pueden gestionar este bien intangible.

\section{Las Mipymes y la gestión del conocimiento}

En la Unión Europea, más del 99\% de las empresas son Mipymes, aportan el 69\% del empleo y el 56\% del PIB. Mientras que en América Latina las micro, pequeñas y medianas empresas son aproximadamente el 99\% de las empresas formales de la región (88.4\% son microempresas), aportan el 61\% del empleo y tienen una participación en el PIB regional del 25\% (Dini y Stumpo, 2018).

En México, del total de establecimientos del sector privado y paraestatal $(4,773,995$ durante 2019), un 99.98\% corresponden a las micros, pequeñas y medianas empresas y aportan el $68.4 \%$ del total de los empleos (INEGI, 2019). Debido a la importancia de las Mipymes de todo el mundo en la economía de la actual sociedad del conocimiento, resulta de especial interés identificar los retos y beneficios que pueden afrontar dichas empresas al gestionar el conocimiento.

Algunos estudios empíricos abordan el tema de la gestión del conocimiento en las micro, pequeñas y medianas empresas identificando retos y beneficios. Por ejemplo, para Lin, Seidel, Shahbazpour y Howell (2013) algunos retos de las pymes de Nueva Zelanda son la falta de recursos financieros, la estructura plana de las organizaciones, la administración a menudo informal, la escasez de recursos humanos calificados y la cultura laboral informal que se convierten en desafíos frente a GC.

En cuanto a los beneficios de la gestión del conocimiento en las Mipymes, estudios como el de García-Tapial (2003) señalan que las pequeñas empresas españolas, que suponen más del 90\% del entramado empresarial de ese país, se benefician a nivel individual como colectivamente al incluir la GC. De esta manera, es importante que identifiquen los conocimientos clave, pues sobre éstos serán capaces de establecer una ventaja competitiva y 
LA GESTIÓN DEL CONOCIMIENTO EN LAS MIPYMES: CASO DE UNA CONSULTORA EN YUCATÁN, MÉXICO.

orientar el desarrollo, mantenimiento o eliminación de actividades, inversiones, estrategias, entre otras acciones.

En el contexto latinoamericano, la situación no es muy diferente. Marulanda y López (2013), observaron en 323 Pymes en ciudades de Colombia que, a pesar de los avances en la implantación de los procesos y del desarrollo de competencias de los colaboradores para la gestión del conocimiento, no se estructuran políticas, programas, planes y proyectos en las organizaciones alrededor de la temática de gestión del conocimiento, además de que estas prácticas no se encuentran enfocadas por completo desde las directrices generales, debido probablemente a su falta de incorporación en la filosofía organizacional.

A su vez, la alta rotación del personal a causa de una falta de formalidad en las relaciones laborales con los trabajadores también es otro desafío. En un estudio realizado en pymes en Ecuador, Arguello (2017) encontró que, por esa razón, una gran cantidad del conocimiento tácito que tienen consigo los colaboradores en forma de experiencias, se pierde, razón por la cual, se incurren a costos de preparación, capacitación y desarrollo de un nuevo elemento.

En México, Gutiérrez-Diez, Sapién y Piñón (2015), mencionan que las Pymes son las más desfavorecidas en el manejo de información para gestionar conocimiento, siendo uno de los principales motivos, la carencia de estrategias y procesos por parte de las organizaciones para realizarlo. Esto lo confirman en el análisis del estudio a 30 Pymes del sector de servicios en la ciudad de Chihuahua en el que se observaron los procesos de gestión de conocimiento. A partir de ello, recomiendan que las actividades de exteriorización sean documentadas.

En un estudio realizado en las Mipymes del sector restaurantero de Bahía de Kino, Sonora, se encontró que la mayoría de las empresas no tienen un plan estratégico formal; asimismo, tanto el personal operativo como el administrativo desconoce su filosofía organizacional y políticas; y no conocen total o parcialmente los aspectos que implican la gestión del conocimiento, por lo que realizan actividades de GC sin estar conscientes, ignorando las ventajas que pudieran obtener de ello (Perez-Soltero, Leal, Barceló y León, 2013).

A pesar de lo anterior, muchas son las Mipymes que se han dado cuenta de la importancia de la implementación de gestión del conocimiento como parte de sus actividades cotidianas para alcanzar el éxito esperado y para crecer. Mayorga, Sánchez y González (2015), realizaron una investigación en 528 Pymes de la zona metropolitana de Guadalajara en la que se concluye que 
LA GESTIÓN DEL CONOCIMIENTO EN LAS MIPYMES: CASO DE UNA CONSULTORA EN YUCATÁN, MÉXICO.

la gestión del conocimiento es un elemento importante para los miembros que integran la organización, debido a que promueve el desarrollo de nuevas capacidades y oportunidades, lo cual ayuda a que las personas desarrollen, estimulen, diseminen, generen e implementen nuevos conocimientos para la creación de pymes competitivas en un mercado globalizado.

Vázquez, Guerrero y Núñez (2014), justifican las razones por las cuales las organizaciones deben usar y fomentar la gestión del conocimiento. La primera, es para crear beneficios exponenciales de conocimiento para los integrantes de la organización que aprenden de éste; de igual forma, apoya en el desarrollo de la capacidad de respuesta oportuna a clientes; una tercera razón es porque construye una confianza en el conocimiento de todos los miembros de la organización y facilita la colaboración para realizar las actividades; además, captura el conocimiento, acción que ayuda a la memoria de la organización. Con base en un estudio realizado, en 418 Pymes manufactureras en los estados de Jalisco, Colima, Querétaro y Aguascalientes, los gerentes de éstas expusieron que entre las bondades de la implementación de la gestión del conocimiento se encontraba el obtener un mejor desempeño financiero.

\section{MATERIAL Y MÉTODO}

Este trabajo se realizó con un enfoque cualitativo mediante el estudio de caso. La investigación cualitativa busca explicar y comprender las interacciones y los significados subjetivos individuales o grupales. Una investigación puede ser valiosa si se realiza en un solo caso (estudio de caso) aun cuando éste sea de pocas personas (Álvarez-Gayou, 2003). Un estudio de caso es una investigación empírica que estudia un fenómeno contemporáneo en profundidad y dentro de su contexto de la vida real, de manera especial cuando los límites entre el fenómeno y el contexto no son claramente evidentes. La investigación del estudio de caso hace frente a la situación técnicamente distintiva en la que habrá muchas más variables de interés que puntos de datos y, como resultado, se basa en múltiples fuentes de evidencia, con datos que deben dirigirse en forma triangular, y como otro resultado se beneficia del desarrollo previo de proposiciones teóricas para orientar la recolección y análisis de datos (Yin, 2009).

El caso que se abordó, fue el de una organización del sector servicios de la ciudad de Mérida, Yucatán, esto es debido a la importancia de dicho sector en la economía del estado, a que la organización es de tamaño pequeño y a que brindó facilidad de acceso a la información. 
LA GESTIÓN DEL CONOCIMIENTO EN LAS MIPYMES: CASO DE UNA CONSULTORA EN YUCATÁN, MÉXICO.

Para la obtención de la información se realizaron entrevistas semiestructuradas al director general y al total de los colaboradores de la organización que son 12, entre personal del área de contabilidad, administración, recepcionista y diligenciero. De la totalidad de los entrevistados, 5 fueron mujeres y 7 hombres. El grado académico de los puestos de dirección y supervisión es de licenciatura. Respecto de los auxiliares uno es estudiante de licenciatura y el resto son estudiantes de carrera técnica. La escolaridad de la persona responsable de recepción es de preparatoria y el encargado de las diligencias cuenta con estudios de secundaria.

Las guías de entrevista se elaboraron con base en la literatura sobre las dimensiones de la gestión del conocimiento y considerando las cinco que se abordaron en la introducción. De igual manera, se hizo uso de la revisión documental y observación como técnicas de investigación complementarias. Las entrevistas fueron grabadas en audio en los casos en que los entrevistados dieron su autorización, y en los demás se tomaron notas. La información obtenida de las observaciones y de la revisión documental se registró en notas en papel.

El análisis de datos se llevó a cabo siguiendo los pasos que menciona Fernández (citado por Urbano, 2016). Todas las entrevistas se transcribieron y las respuestas dadas por los participantes se codificaron en función de las características de las dimensiones de GC. En la codificación de la información se identificaron las opiniones respecto de las prácticas de adquisición, almacenamiento, compartición, aplicación y protección. Para la integración de la información se realizó una comparación de los códigos que se repetían con mayor frecuencia o aquellas que se consideraron de mayor relevancia entre las opiniones de cada integrante de la organización y la respectiva dimensión a la que éstas correspondían. El análisis se complementó con los resultados de la revisión documental y la observación de la operación diaria de la organización.

\section{RESULTADOS}

\section{Adquisición}

Se encontró que el conocimiento en la organización se obtiene principalmente de cursos de capacitación internos y externos, asesoría y colaboraciones con expertos, consultas vía online de materiales y documentos que se encuentran en internet, de la experiencia de cada persona que labora en la consultora, de la aplicación de benchmarking y del uso de revistas. 
LA GESTIÓN DEL CONOCIMIENTO EN LAS MIPYMES: CASO DE UNA CONSULTORA EN YUCATÁN, MÉXICO.

Con respecto a las capacitaciones, los supervisores del área de contabilidad acuden de manera presencial y virtual a cursos, conferencias, paneles y pláticas sobre temas empresariales que imparte la Secretaría de Administración Tributaria (SAT) y otras dependencias, incluyendo a la Cámara Nacional de Comercio, Servicios y Turismo de Mérida (CANACO Servytur Mérida), la Cámara Nacional de la Industria de Transformación (CANACINTRA) y el Instituto Mexicano de la Propiedad Industrial (IMPI) de manera gratuita, en los que actualizan sus conocimientos y despejan dudas sobre temas de interés. Adicional a las capacitaciones gratuitas, los supervisores complementan la obtención de su conocimiento con cursos pagados por la consultora.

Actualmente, la práctica de adquisición de conocimiento contemplada para los auxiliares contables son las capacitaciones internas. Sin embargo, mencionan que a ellos también les gustaría acudir a los cursos gratuitos, ya que consideran que eso les permitiría escuchar opiniones de expertos con amplia experiencia y les haría sentirse parte de la organización.

El área de administración también acude a cursos gratuitos, a diferencia del área contable, estos son impartidos por el Instituto Mexicano del Seguro Social (IMSS), el Instituto del Fondo Nacional de la Vivienda para los Trabajadores (INFONAVIT), el INEGI entre otros organismos y por lo general se realizan de manera virtual.

El personal de ambas áreas (administración y contabilidad) hace uso de llamadas a la asistencia en línea que tienen los organismos anteriores y que les proporciona orientación sobre sus dudas, además del chat con el que los portales de dichas dependencias cuentan. Los foros en internet y grupos son otro medio por el que se obtiene información que al corroborar con fundamentos legales se convierten en conocimiento. Además, consultan libros, páginas web, leyes, reglamentos, etc. que se encuentran de manera digital y que contribuyen para otorgarles una mejor comprensión de cuestiones que tienen que ver con el desarrollo de sus actividades.

En la recepción, las instrucciones y capacitaciones provienen del director general con respecto a determinados formatos, del área de contabilidad en cuanto a temas relacionados a la facturación y del área de administración en cuestiones de cobranza. Asimismo, la encargada de recepción se ha apoyado en cursos digitales:

Cuando tengo un tiempecito libre me pongo a ver tutoriales en YouTube o de alguna otra red social que me permita ser más rápida y aprender a manejar mejor los programas que 
LA GESTIÓN DEL CONOCIMIENTO EN LAS MIPYMES: CASO DE UNA CONSULTORA EN YUCATÁN, MÉXICO.

utilizo o para aprender a instalarlos; sin embargo, tengo que esconderme un poco o puede pensar el jefe que me encuentro viendo videos porque no tengo nada que hacer...

El personal de ambas áreas también obtiene conocimientos mediante la suscripción en revistas de circulación digital y gratuita, como son: Expansión, Alto Nivel y Forbes. Los supervisores contables se encuentran inscritos a la revista Contaduría Pública publicada por el Instituto Mexicano de Contadores Públicos (IMCP), el resto del personal no cuenta con ninguna suscripción ni menciona haber leído alguna. Para la organización es importante realizar estudios del entorno para conocer el mercado, el director general señaló que él realiza un estudio de benchmarking en el que compara y toma de referencia a otras consultoras de su mismo tamaño o más grandes y mejor posicionadas; sin embargo, no se encontró evidencia documental de esto.

\section{Almacenamiento}

La organización cuenta con una intranet que se utiliza para almacenar archivos de trabajo, material de referencia (leyes, artículos o notas en los que se basan para realizar cálculos y formatos), proyectos, certificaciones de cursos de capacitación a los que acude el personal a nombre de la consultora sobre temas de negocio, además de expedientes, todos ellos compartidos exclusivamente por los miembros de la organización. Pese a los beneficios que la intranet ofrece, los colaboradores consideran que ésta podría ser mejor aprovechada si no hubiera falta de control de los documentos que se encuentran resguardados y dificultades para adaptarse a las rutas que deben seguir para encontrar determinados documentos en las diversas carpetas digitales.

Algunos de los documentos electrónicos más importantes dentro de la intranet que se mencionaron son la guía telefónica de los clientes y proveedores, que contiene información sobre los nombres de las empresas, datos de contacto, teléfonos celulares y fijos, correo electrónico, dirección y otros datos de facturación, así como fechas de cumpleaños. Asimismo, los colaboradores destacaron que es importante el resguardo de información personal de los clientes.

Uno de los supervisores, explica una forma que utilizan para almacenar sus experiencias sobre trabajos previos: 
LA GESTIÓN DEL CONOCIMIENTO EN LAS MIPYMES: CASO DE UNA CONSULTORA EN YUCATÁN, MÉXICO.

Al finalizar proyectos con cualquier cliente, siempre incluimos a su carpeta una ficha que contiene una descripción sobre la forma de trabajar con ellos, ya que existen clientes a los que no les gusta que los llamemos a su celular o hay quienes prefieren que las reuniones de trabajo sean programadas con semanas de anticipación, lo cual nos indica cómo debemos proyectar nuestro plan de trabajo.

A pesar de que la organización no cuenta con un sistema Enterprise Resource Planning (ERP), tiene programas que apoyan a los colaboradores con las funciones que realizan, ejemplo de ellos son el software contable y de facturación electrónica CONTPAQ, el sistema de nómina integral ASPEL NOI, el programa estadístico informático Statistical Package for the Social Sciences (SPSS) además del Microsoft Office y otros programas gratuitos con los cuales la organización genera datos e información para la toma de decisiones y la generación del conocimiento además de almacenar información.

De acuerdo con la revisión de documentos realizados, no se encontraron manuales redactados en la consultora. Esto podría estar ocasionando que los colaboradores no tengan un conocimiento claro de sus funciones dentro de la organización y se afectaría el proceso de la comunicación, integración y desarrollo de sus miembros.

\section{Compartición}

Para fomentar la compartición de conocimiento, la dirección general pide a las personas encargadas de cada área que capaciten a los puestos de nuevo ingreso. Estas capacitaciones son aprovechadas por los auxiliares que por lo general se encuentran ávidos por adquirir conocimientos. Por otro lado, los encargados de recepción y diligencias no encuentran provechoso que todos los puestos les enseñen a ellos cómo realizar determinado trabajo porque consideran que los mandos superiores esperan que los trabajos enseñados sean los primeros que realicen.

Los auxiliares obtienen conocimiento de sus supervisores tomando apuntes en cuadernos, mismos que utilizan posteriormente cuando tienen dudas al momento de realizar el trabajo. Al concluir su relación laboral con la consultora, los auxiliares pueden llevarse dichos cuadernos. Los auxiliares se comparten conocimiento entre ellos, ya sea a través de apuntes, o se etiquetan en alguna nota de interés como noticias de temas financieros y empresariales o se envían información en redes sociales como grupos de WhatsApp y Facebook, mismas que crearon por decisión propia. Uno de los auxiliares comenta al respecto: 
LA GESTIÓN DEL CONOCIMIENTO EN LAS MIPYMES: CASO DE UNA CONSULTORA EN YUCATÁN, MÉXICO.

En mi caso tengo dos grupos de WhatsApp, en uno se encuentra todo mi departamento (contabilidad) y en el otro sólo mis compañeros (auxiliares) sin que se incluyan los supervisores, el primero lo utilizan los supervisores cuando les urge que les mandemos algún formato o cuando quieren que leamos alguna noticia y el segundo lo utilizamos nosotros (auxiliares) para apoyarnos.

Otro medio utilizado es el correo electrónico, ya que a través de él se envían y reciben indicaciones para la realización de alguna actividad, se adjuntan archivos, se envían hipervínculos de información, retroalimentaciones, invitaciones para cursos de capacitación, entre otros.

La organización no cuenta con una estrategia formal como lo sería un mecanismo de incentivos o la creación de espacios físicos o virtuales compartidos que inspiren a interacciones constructivas para motivar a que sus colaboradores compartan sus experiencias y mejores prácticas. Los colaboradores mencionaron que es por iniciativa propia que comparten sus experiencias y conocimientos con sus compañeros usando mecanismos informales como las redes sociales, cuando se reúnen a festejar algún cumpleaños del personal o conmemorar una fecha importante.

La comunicación es una debilidad en la consultora debido a que el personal por lo general no comparte los asuntos que puedan estar afectando su trabajo como el de recibir indicaciones por diferentes personas y que todas ellas requieran sus trabajos en calidad de urgentes. Adicional a lo anterior, algunos colaboradores señalaron que la dirección general no comunica tareas que afectan a otras áreas lo que crea duplicidad u omisión en ellas, generalmente provocado porque los puestos desconocen el alcance de sus responsabilidades.

\section{Aplicación}

Los colaboradores señalaron que aplican su conocimiento durante el desarrollo de los servicios que ofrece la organización, por ejemplo, uno de los supervisores comenta:

Al laborar en una organización de servicios como es la consultora, los clientes esperan que el trabajo que les realicemos les proporcione herramientas que permitan elevar su nivel de competitividad en el mercado, para ello nosotros tenemos que profundizar en aspectos generales e inclusive específicos de la empresa a la que le diseñamos un plan de trabajo, esos aspectos incluyen desde conocer a qué se dedican, sus procesos, organigrama, estados financieros, metas, objetivos, expectativas, hasta sus conflictos 
LA GESTIÓN DEL CONOCIMIENTO EN LAS MIPYMES: CASO DE UNA CONSULTORA EN YUCATÁN, MÉXICO.

internos. Una vez que hayamos conocido lo anterior, nos toca a nosotros aplicar el conocimiento que hemos adquirido en cursos de capacitación y experiencias obtenidas para lograr realizar el trabajo que nos encomendaron.

De esta manera cada proyecto o servicio que ofrecen se convierte en nuevo conocimiento que pueden aplicar en servicios posteriores, para ello la experiencia previa y actual adquiere un valor importante en este proceso. Por su parte los auxiliares consideran que realizan la aplicación del conocimiento que adquieren después de las capacitaciones internas, así como al momento de llevar a la práctica la teoría que les enseñan en sus escuelas. Algunos colaboradores mencionaron que aplican conocimientos adquiridos por experiencias propias, así lo comenta el encargado de las diligencias en la organización:

Yo no tengo capacitaciones, lo que tengo de conocimiento lo obtengo gracias a las situaciones a las que me expone mi puesto y lo aplico porque aprendo de esa experiencia o porque ya son situaciones rutinarias como el saber cuánto de gasolina voy a necesitar para ir a algún lugar.

Los supervisores mencionan que en la consultora no existen mecanismos formales de reconocimiento para la aplicación de conocimiento, sin embargo, ellos se encargan de fomentar que los auxiliares aporten sus ideas y tengan libertad de realizar modificaciones a las ideas propuestas. A su vez, los auxiliares consideran que su conocimiento se aplica cuando los supervisores les permiten realizar mejoras en los formatos y trabajos previa autorización, al respecto, uno de los auxiliares contables comenta:

Una de las razones por las que decidí realizar mis prácticas en este lugar es porque me permiten incorporar mis ideas, los supervisores no son cerrados y si tú vas y les dices por ejemplo que conoces una fórmula en Excel que permite hacer un cálculo más rápido, lo revisan y si les convence lo aprueban.

Con la apertura e incorporación de nuevas ideas, se ha logrado que los proyectos cada vez tengan nuevos y mejores elementos y se han reducido los tiempos de realización de algunas actividades.

\section{Protección}

La consultora maneja información valiosa y confidencial de sus clientes. Para proteger esta información los colaboradores firman una carta de confidencialidad al ingresar a laborar en la organización. En el proceso de recepción y entrega de documentación de los clientes, se 
LA GESTIÓN DEL CONOCIMIENTO EN LAS MIPYMES: CASO DE UNA CONSULTORA EN YUCATÁN, MÉXICO.

realizan dos cartas en la que se señala la información que la organización se encuentra recibiendo o entregando, una de las cartas es para la seguridad del cliente y la otra es para el resguardo de la consultora.

Otra protección con la que cuenta la organización es en cuanto a su marca, ya que esta se encuentra registrada en el IMPI. Un punto débil en cuanto a la exposición de la información es que el acceso a ésta la tienen todos los colaboradores a través de la intranet, ya que no se cuentan con restricciones en el sistema, lo cual trae como consecuencia que cualquier persona pueda borrar alguna información valiosa o enviarla por equivocación a otra.

\section{Tabla 1}

\section{Prácticas de gestión del conocimiento identificadas}

Dimensiones Prácticas llevadas a cabo por los integrantes de la consultora

Adquisición Cursos de capacitación internos y externos, asesoría y colaboraciones con expertos, consultas vía online de materiales y documentos que se encuentran en internet, experiencia del personal, revistas.

Almacenamiento Intranet, software (CONTPAQ, ASPEL NOI, SPSS, Microsoft Office), fichas sobre experiencias de trabajos a clientes.

Compartición Capacitaciones internas, cuadernos con apuntes, Facebook, WhatsApp, correo electrónico, pláticas informales.

Aplicación Durante el desarrollo de los servicios que ofrece la organización y después de capacitaciones internas, se observa en mejoras de formatos, trabajos y reducción de tiempos en la realización de proyectos.

Protección Cartas de confidencialidad, cartas de entrega y devolución de documentación, registro de marca.

Fuente: Elaboración propia con datos obtenidos del trabajo de campo.

\section{DISCUSIÓN Y CONCLUSIONES}

Con base en los resultados puede concluirse que la organización no tiene una estrategia formal de gestión del conocimiento; sin embargo, se identificó que llevan a cabo ciertas prácticas relacionadas con las cinco dimensiones. Han trabajado más en prácticas relacionadas con la adquisición y menos en prácticas relativas a la compartición y protección del conocimiento.

La adquisición del conocimiento en la consultora proviene principalmente de dos estrategias como señala Riesco (2014): a) la compra del mercado o alquiler del conocimiento en el mercado o de las personas externas a la organización b) creación dentro de la misma 
LA GESTIÓN DEL CONOCIMIENTO EN LAS MIPYMES: CASO DE UNA CONSULTORA EN YUCATÁN, MÉXICO.

organización. Esto es debido a que participan en cursos de capacitación externos, asesoría y colaboraciones con expertos, así como de la consulta vía online de materiales y documentos que se encuentran en internet. Respecto de la segunda estrategia, al aprovechar la experiencia de cada persona que labora en la organización y mediante las capacitaciones internas impartidas.

El almacenamiento se ve reflejado en la intranet que la consultora utiliza para almacenar archivos de trabajo, los cuales son aprovechados por los miembros de la organización. Sin embargo, esta intranet requiere de orden y de un mejor control de acceso a la información para retener únicamente el conocimiento valioso y necesario como señala Riesco (2014).

Con respecto a la compartición del conocimiento esta se da mediante mecanismos formales principalmente a través de capacitaciones, sin embargo, también existen medios informales como las reuniones o redes sociales (Pérez-Montoro, 2008).

La aplicación del conocimiento se observa durante el desarrollo de los servicios que ofrece la organización por medio de la experiencia previa y actual, así como después de las capacitaciones internas y al momento de llevar a la práctica la teoría que les enseñan en sus escuelas en el caso de los estudiantes. Como señala, Wiig (2004), algunas aplicaciones de conocimiento se ven reflejadas en las mejoras de los proyectos para sus clientes.

Aunque se tienen algunas prácticas de protección, se debe considerar lo que propone Kerschberg (2001), cuando señala que deben establecerse controles de acceso que se puede reflejar en las cartas de confidencialidad y políticas de seguridad para proteger la base de conocimiento y la propiedad intelectual.

En cuanto a los retos, se pudo confirmar lo señalado por Marulanda y López (2013), respecto de que en las Mipymes no se estructuran formalmente políticas, programas, planes y proyectos alrededor de la temática de gestión del conocimiento a pesar de los avances en la implantación de los procesos y del desarrollo competencias de los colaboradores, esto debido probablemente a su falta de incorporación en la filosofía organizacional. También coincide con lo que menciona Pérez Soltero et al. (2013) cuando refiere que los colaboradores que no conocen el concepto total o parcial, así como los procesos de gestión de conocimiento, realizan las actividades relacionadas a ello, sin estar conscientes, ignorando las ventajas que pudieran obtener. Debido a la rotación de los auxiliares contables se pierde gran cantidad de 
LA GESTIÓN DEL CONOCIMIENTO EN LAS MIPYMES: CASO DE UNA CONSULTORA EN YUCATÁN, MÉXICO.

conocimiento tácito en forma de experiencias que tienen consigo, lo que provoca gastos de selección, capacitación y desarrollo de un nuevo elemento (Arguello, 2017). Otro reto que presenta la consultora, es señalado por Gutiérrez-Diez et al. (2015), cuando menciona que las pymes son las más desfavorecidas en el manejo de la información para gestionar conocimiento, esto debido a la carencia de estrategias y procesos para llevarlo a cabo.

En cuanto a los beneficios derivados de las prácticas de gestión del conocimiento que actualmente lleva a cabo la consultora, se encuentra el desarrollo de la capacidad de respuesta oportuna a clientes (Vázquez et al., 2014), la capacitación y formación continua del personal (García-Tapial, 2003) y el desarrollo de nuevas capacidades y oportunidades, lo cual apoya a que los miembros de la organización implementan mejoras en sus servicios (Mayorga et al., 2015).

Este trabajo permitió identificar oportunidades de mejora para cada una de las cinco dimensiones, con respecto a la adquisición, es importante diseñar estrategias para evitar la rotación del personal, así como fomentar el uso de las redes sociales para la adquisición del conocimiento y la realización de estudios de mercado que permitan identificar las necesidades de los clientes para ofrecer servicios diferenciados. En cuanto al almacenamiento se requieren estrategias que permitan mantener el orden y codificación de la información que se encuentra en la intranet, así como el desarrollo de manuales de organización y sistematización de sus bases de datos. Con respecto a la compartición se recomienda el diseño de estrategias formales que promuevan esta práctica como el establecimiento de incentivos por fungir como asesor o apoyo de los compañeros o la conformación de equipos de trabajo, así como el fomento del diálogo de los mandos bajos hacia los mandos medios o superiores y viceversa. En lo que respecta a la aplicación del conocimiento, se recomienda el establecimiento de mecanismos formales de reconocimiento para esta práctica y en cuanto a la protección se requieren estrategias que permitan un acceso controlado a la información de clientes. 
LA GESTIÓN DEL CONOCIMIENTO EN LAS MIPYMES: CASO DE UNA CONSULTORA EN YUCATÁN, MÉXICO.

\section{REFERENCIAS BIBLIOGRÁFICAS}

Álvarez-Gayou, J. (2003). Cómo hacer investigación cualitativa. Fundamentos y metodología. México: Editorial Paidós Mexicana, S.A.

Angulo, E. y Negrón, M. (2008). Modelo holístico para la gestión del conocimiento. Revista Negotium, 11(4), 38-51.

Arboníes, A. (2006). Conocimiento para innovar. España: Ediciones Díaz de Santos, S.A.

Arguello, C. (2017). Desarrollo de un modelo de gestión del conocimiento para las PYMES del sector textil de la ciudad de Riobamba. Industrial Data, 20(2), 79-86.

Caraballo, Y. Mesa, D. y Herrera, J. (2009). Herramientas de gestión del conocimiento: convergencias hacia un aprendizaje organizacional. Revista Cubana de Ciencias Agrícola, 43(1), 1-13.

Davenport, T. y Prusak, L. (2000). Working knowledge: how organizations manage what they know. Ubiquity, ACM. Recuperado de https://ubiquity.acm.org/article.cfm?id=348775

Diario Oficial de la Federación (DOF, 2009). ACUERDO por el que se establece la estratificación de las micro, pequeñas y medianas empresas. Recuperado de http://dof.gob.mx/nota_detalle.php?codigo=5096849\&fecha=30/06/2009

Dini, M. y Stumpo, G. (2018). Mipymes en América Latina. Un frágil desempeño y nuevos desafíos para las políticas de fomento. Santiago: Comisión Económica para América Latina y el Caribe (CEPAL).

Durán, P. (2017). Microempresas, PyMEs y Objetivos de Desarrollo Sostenible. Recuperado de http://www.onu.org.mx/microempresas-pymes-y-objetivos-de-desarrollo-sostenible/

García-Tapial, J. (mayo/junio, 2003). La gestión del conocimiento es ya una realidad para la empresa española. El profesional de la información, 12(3), 206-213.

Gutiérrez-Diez, M., Sapién, A. y Piñón, L. (2015). Gestión de conocimiento en PyME del sector servicios en la ciudad de Chihuahua. Nova Scientia, 7(15), 499-513.

INEGI (2019). Censos económicos 2019. Recuperado de http://internet.contenidos.inegi.org.mx/contenidos/Productos/prod_serv/contenidos/espan ol/bvinegi/productos/nueva_estruc/702825192983.pdf 
LA GESTIÓN DEL CONOCIMIENTO EN LAS MIPYMES: CASO DE UNA CONSULTORA EN YUCATÁN, MÉXICO.

Kerschberg, L. (2001). Knowledge Management in Heterogeneous DataWarehouse Environments. Recuperado

de https://www.academia.edu/539290/Knowledge_Management_In_Heterogeneous_Data_ Warehouse_Environments

Lin, I., Seidel, R., Shahbazpour, M. y Howell, D. (2013). Knowledge Management in Small and Medium-sized Enterprises: A New Zealand Focus. New Zealand journal of applied business research, 11(2), 1-21.

Maldonado G., Martínez, M. y García, R. (enero/julio, 2012). Gestión del conocimiento y crecimiento en la Pyme manufacturera de Aguascalientes (México). Cuadernos de Investigación. Universidad del Valle, 28(47), 25-36.

Marulanda, C. y López, M. (febrero/mayo, 2013). La gestión del conocimiento en las PYMES de Colombia. Revista Virtual Universidad Católica del Norte, (38), 158-170.

Mayorga, P., Sánchez, J. y González, E. (noviembre, 2015). Efectos de las estrategias de la gestión del conocimiento en la competitividad de las pymes. Red Internacional de Investigadores en Competitividad Memoria del IX Congreso, 1728-1749.

Mul, J. y Ojeda, R. (2014). Análisis de la gestión del conocimiento en empresas con actividades de innovación en Yucatán. XIX Congreso Internacional de Contaduría, Administración e Informática. UNAM. Recuperado de http://premio.investiga.fca.unam.mx/docs/ponencias/2014/2.1.pdf

Ramírez, J., Mercado, J. y Barbosa, M. (2012). Contexto actual de la protección del conocimiento: propiedad intelectual en la Universidad Autónoma del Estado de México. XVII Congreso Internacional de Contaduría, Administración e Informática. UNAM. Recuperado de: http://congreso.investiga.fca.unam.mx/docs/xvii/docs/D04.pdf

Nonaka, I. (1994). A dynamic theory of organizational knowledge creation. Organization Science, 5(1), 14-37.

Pérez-Montoro, M. (2008). Gestión del conocimiento en las organizaciones. Fundamentos, metodología y praxis. España: Ediciones Trea, S.L. 
LA GESTIÓN DEL CONOCIMIENTO EN LAS MIPYMES: CASO DE UNA CONSULTORA EN YUCATÁN, MÉXICO.

Perez-Soltero, A., Leal, V., Barceló, M. y León, J. (2013). Un diagnóstico de la gestión del conocimiento en las pymes del sector restaurantero para identificar áreas de mejora en sus procesos productivos. Intangible Capital, 9(1), 153-183.

Ramírez, J., Mercado, J. y Barbosa, M. (2012). Contexto actual de la protección del conocimiento: propiedad intelectual en la Universidad Autónoma del Estado de México. XVII Congreso Internacional de Contaduría, Administración e Informática. UNAM. Recuperado de http://congreso.investiga.fca.unam.mx/docs/xvii/docs/D04.pdf

Riesco, M. (2004). Gestión del conocimiento en ámbitos empresariales: "modelo integradosituacional" desde una perspectiva social y tecnológica (Tesis doctoral, Universidad Pontificia de Salamanca). Recuperado de https://summa.upsa.es/viewer.vm?id=14258\&amp;view=main\&amp;lang=es

Rodríguez, D. (2006). Modelos para la creación y gestión del conocimiento: una aproximación teórica. Educar, (37), 25-39.

Sánchez, C. y Ríos, H. (2011). La economía del conocimiento como base del crecimiento económico en México. Enl@ce: Revista Venezolana de Información, Tecnología y Conocimiento, 8(2), 43-60.

Urbano, P. (2016). Análisis de datos cualitativos. Revista Fedumar Pedagogía y Educación, 3(1), 113-126.

Vázquez, G., Guerrero, J. y Núñez, T. (enero/junio, 2014). Gestión de conocimiento, capital intelectual y competitividad en pymes manufactureras en México. RETOS. Revista de Ciencias de la Administración y Economía, 4(7), 28-43.

Wiig, K. (2004). People-Focused Knowledge Management. How Effective Decision Making Leads to Corporate Success. USA: Elsevier, Inc.

Yin, R. (2009). Case Study Research. Design and Methods. California: SAGE Inc. 